\title{
Entretien avec Tahar ben Jelloun
}

(Propos recueillis par Francesca DAINESE, Salon du livre de Paris, dimanche 23 mars 2014)

Tahar Ben Jelloun est un écrivain et poète marocain de langue française très connu du grand public en France et en Europe grâce à son roman La nuit sacrée (Prix Goncourt, 1987), suite de L'enfant de sable. Après avoir fréquenté une école primaire bilingue arabo-francophone, le lycée français de Tanger et l'Université de Philosophie de Rabat, il est devenu Docteur en psychiatrie sociale en 1975, à Paris, où il a débuté sa carrière d'écrivain. Aujourd'hui Tahar Ben Jelloun est l'auteur de plusieurs romans, mais aussi d'essais pédagogiques, dont Le racisme expliqué à ma fille, paru en 1997. En 2008, il succède à François Nourissier comme membre de l'Académie Goncourt. Il a accepté cette entrevue parce que je ne lui ai pas demandé de parler de ses œuvres, ni de L'ablation, son dernier roman, mais d'un sujet pour lequel il s'est engagé tout au long de sa vie : la Francophonie.

En parlant de francophonie, qu'il y a-t-il de nouveau à dire ? Une telle « étiquette »a-telle encore du sens dans la littérature française aujourd'hui ?

Je vous donne tout d'abord un exemple pour m'expliquer : il y a quelque temps que j'ai posé la question à Alain Robbe-Grillet, il m’a répondu simplement : « je ne sais pas, je suis français, je ne suis pas francophone ». Cela pose un problème : francophone pour un Français veut dire automatiquement : il n'est pas français. Et donc que les écrivains français ne sont pas francophones. Cette situation est tout à fait différente pour les écrivains anglais : il y a beaucoup d'écrivains d'origine asiatique, pakistanaise... qui vivent à Londres, ou n'importe où, et écrivent en anglais, mais ils ne sont pas considérés différemment comme c'est le cas en France. Moi j'écris en français, c'est la langue que j'ai apprise à l'école, je ne dois pas me justifier ! Cette sorte de méfiance n'importe pas pour moi, mais c'est pertinent d'observer qu'elle existe et qu'il faut la combattre.

\section{Est-ce le motif pour lequel vous avez accepté cet entretien ?}

La question de la francophonie a toujours été primordiale pour moi. J'en ai parlé dans beaucoup de retransmissions télévisées, et dans les journaux aussi. C'est comme défendre un droit. En plus je n'aime pas parler de mes livres, je n'aime pas me substituer aux critiques, décoder des signes soi-disant cachés entre les lignes auxquels, souvent, je n’ai même pas pensé ! Je préfère parler de ce qui nous entoure, en tant qu'écrivains, en tant que citoyens.

Selon vous pourquoi il y a cette différence entre France et Angleterre au niveau de la reconnaissance littéraire pour les auteurs d'origine coloniale?

La France a un rapport particulier avec ses anciennes colonies, qui n'est pas tout à fait apaisé. Par exemple la Guerre d'Algérie, terminée en 1962, il y a donc de ça un demi-siècle, n'est pas quelque chose que l'Histoire a encore digéré. 


\section{Vous écrivez en français, pourquoi ? Qu'est-ce que cela signifie pour vous ?}

J'ai eu très vite une éducation bilingue, pour moi écrire en français était un plus qui me permettait d'exprimer en français des choses que je pouvais dire (et ne pas dire) en arabe. Au début c'était aussi une façon de le cacher à mes parents ! Mais une fois que j'ai commencé à écrire en français, je ne me suis jamais arrêté. Maintenant j’ai du mal à écrire en arabe, écrire un livre entier en arabe pour moi est impossible. En effet c'est une langue magnifique, très riche, mais aussi très difficile. En France la langue de Racine, de Rabelais est un exemple suprême de belle langue, en Angleterre on trouve Shakespeare, ou Dante en Italie ... mais en arabe on n'a pas un écrivain représentatif. Le plus grand poète arabe aujourd'hui ne maîtrise pas à $100 \%$ la langue. Par exemple le grand Mahmoud Darwisc, palestinien, excelle, mais on peut considérer seulement le Coran, encore, le vrai représentant de la langue arabe. Il est très bien écrit, très fort, comme certain textes de la Bible.

\section{La langue française est-elle plus laïque?}

Oui, et en même temps on peut se permettre des écarts. Je ne suis pas capable de le faire en arabe, je ne suis pas investi dans la langue arabe. En français je me suis toujours senti plus libre, même pour créer, imaginer, maltraiter la langue.

Selon Pierre Assouline, les écrivains francophones sont aujourd'hui les seuls capables d'innover, d'irriguer la langue, de l'enrichir. Qu'en pensez-vous ?

Il y a des écrivains vraiment novateurs, du Congo, de la Guadeloupe, de la Martinique... Patrick Chamoiseau, par exemple, il écrit en créole, il créolise la langue français, il l'enrichit fortement. D'autre part, c'est vrai que personnellement quand je l'ai lu j'ai eu besoin d'un dictionnaire, j'ai eu du mal à comprendre. Trop d'innovation. J'ai rencontré Chamoiseau au Maroc il y a trois semaines. En parlant, j'ai senti chez lui la recherche de la difficulté, il aime bien aller un peu plus loin que les écrivains classiques.

Votre langue est simple, élégante, il y a plutôt une recherche de signification qu'un simple enrichissement de mots.

J'écris en français mais je parle d'une société qui n'est pas la société française, peut-être que c'est pour cela. La tradition marocaine, ou arabe, favorise cette approche de la langue, c'est-à-dire : on donne une importance thématique à nos œuvres. Amin Maalouf, libanais, ou Boualem Sansal, algérien, utilisent un langage simple aussi. Le public aime notre littérature parce qu'il trouve dedans quelque chose qu'il cherche au niveau thématique. La langue française a cet avantage d'être utilisée par des écrivains qui viennent d'ailleurs et cet ailleurs est intéressant. 
Comment pensez-vous être perçu à travers le monde ? Quelle image renvoyez-vous ? Que cherche votre public en lisant vos ouvres?

Dans un premier temps, peut-être qu'il cherche à être dépaysé. En même temps, moi je ne fais pas de la littérature exotique ou folklorique, cela n'a pas de sens pour moi. Mais même quand je raconte une histoire moderne, comme le Bonheur conjugal, que j'ai écrit l'année dernière (le récit parle d'un thème universel, mais installé au Maroc, avec le conflit culturel et sociologique de la société marocaine), on a vu que le livre a eu du succès et a été beaucoup traduit en italien, en allemand... Cela veut dire que ce n'est pas qu'une histoire marocaine qui a été appréciée, mais quelque chose qui concerne tout le monde. Cela dépend aussi de l'écrivain, je ne sais pas comment je suis perçu, mais j'écris depuis quarante ans et je suis sûr que, si je n'avais pas un public qui suive ce genre de littérature, je n'aurais pas pu continuer. Je ne considère pas qu'on puisse écrire si on n'a pas de public. Écrire pour soi dans un petit coin... non. Moi, j'écris parce que je sens qu'il y a un public qui existe.

Vous êtes bien connu et bien traduit dans plusieurs pays. On dirait que vous avez eu un sort différent des autres écrivains francophones. Qu'est-ce qui a changé dans votre cas ?

Le prix Goncourt. C'est la spécialité française, c'est unique dans le monde. Il n'y a pas un prix littéraire qui mobilise autant de média et autant d'intérêt dans le monde. Venez chez Drouant, en novembre prochain. Si vous arrivez à passer, ce serait pas mal ! C'est un événement qui se traduit par un intérêt international. Le prix Goncourt traduit en quarante-cinq langues, c'est énorme. Cela ouvre des perspectives extraordinaires. De toute façon, parlant traduction, on peut dire que la France est un pays où on traduit beaucoup, comme l'Italie. En Amérique seulement 3\% de la production publiée sont des livres étrangers. C'est rien. En France, c'est presque $40 \%$ et, en Italie, c'est pareil. Nous sommes intéressés à ce qu'il se passe ailleurs, c'est une qualité, la traduction est un signe d'ouverture, de curiosité vers l'autre. C'est un mérite des maisons d'édition. Mais on traduit ce qui marche, les best-sellers. On va regarder les statistiques, le succès va au succès.

La valeur sociale et médiatique d'un tel prix change selon vous considérablement l'intérêt des éditeurs étrangers pour certains auteurs?

Tout à fait, après un Prix comme le Goncourt ou d'autres prix, les maisons d'édition achètent les droits français et font traduire, mais c'est vrai aussi qu'il y a un investissement de la part de l'auteur. Dans mon cas, après le Goncourt, par exemple en Italie, j'ai été publié par les grandes maisons d'édition Bompiani et Einaudi, mais je suis allé une centaine de fois présenter mes livres, faire des rencontres avec le public ; publier ici [ndlr : en Italie] est devenu une affaire aussi importante qu'en France. Après, il faut dire aussi que la thématique d'un certain livre influe au niveau de la diffusion : en Italie, je suis devenu très populaire après le livre sur le racisme, ce qui n'est 
pas représentatif de ma production littéraire. Mais surtout je suis allé dans des dizaines d'écoles dans le monde, j’aime aller dans les écoles.

L’intérêt pour les écoles est très important. Selon vous, quel est le rôle de la littérature dans la société d'aujourd'hui ?

Les gens veulent savoir ce qui se passe dans la société. Mes livres pédagogiques, comme je l'ai déjà dit, ne sont pas représentatifs de mon travail littéraire, mais ils sont importants parce que il y a une carence, il y a un vide, les gens ne savent pas ce qui les entoure. Ils ont besoin d'apprendre ce qu'il se passe. Par exemple, encore à propos de l'Italie, qui peut expliquer la vague d'immigration venue du Maghreb et de l'Afrique en général ? Cela fait désormais vingt-cinq ans, mais personne n'était là pour parler de la situation. Il y avait seulement la Lega Nord faisant le travail pour le racisme. D'autre part, c'est vrai que le rôle de la littérature c'est aussi de raconter des histoires. Quand je suis allé au Mexique, je n'ai pas acheté de guide. J'ai acheté Carlos Huante ; Borges, Ernesto Sabato avant de partir pour l'Argentine. Un romancier est le meilleur traducteur de la société : je pense que c'est important de faire jouer à la littérature un rôle informatif sur la société. On comprend mieux l'Italie quand on lit Moravia. Quand j'ai beaucoup travaillé sur la Sicile, j'ai lu Sciascia. Même quand je lisais Italo Calvino, c'était une façon imaginaire de voir l'Italie. Pour moi, la littérature est une géographie de l'imaginaire.

On ne parle pas d'un engagement idéologique, mais dans quelle mesure la littérature, pour vous, est-il un témoignage actif d'un moment historique ? Quel est le rôle de l'écrivain ?

L'écrivain ce n'est pas uniquement quelqu'un qui écrit des livres, c'est aussi quelqu'un qui intervient en tant que citoyen dans la vie. C'est pour cela que je bouge beaucoup, je considère qu'il faut aller vers les gens. Le livre sur le racisme m’a donné un rôle public, et cela a continué.

\section{Le rôle de l'écrivain a-t-il changé ces dernières années ?}

Il y a des écrivains qui ont fait la différence, comme Zola, Sartre... Camus surtout, extrêmement préoccupé par son pays, par l'histoire. Il a écrit des textes magnifiques, il est un des écrivains majeurs du vingtième siècle, encore aujourd'hui lu et commenté. Gide, plus préoccupé par sa personne, sa philosophie, n'était pas un écrivain en contact politique avec la société. Les nourritures terrestres était un roman très fort, mais pas engagé. L'étranger, ou La chute de Camus sont eux des écrits universels. Pour moi, un écrivain c'est un citoyen. Il peut avoir des opinions politiques, il peut appartenir à un parti politique. Ce n'est pas mon cas, je suis très préoccupé par la condition humaine en général, les droits de l'homme, les droits de la femme, l'immigration, parce que ça m'intéresse, je ressens le besoin de faire de la littérature réelle, dramatique. L'engagement ce n'est pas la littérature idéologique, moi je la déteste. Je reste préoccupé par la condition des gens. 


\section{Qu'est-ce que Paris représente pour vous?}

J'habite ici par commodité, mon tourné vers le Maroc, vers le monde arabe. Les Français n'ont pas besoin de moi pour écrire sur leur pays, ils ont assez d'écrivains. J'ai publié des textes sur la France plutôt critiques, à vrai dire, sur l'hospitalité française. Ils ont été très mal reçus. C'était ironique, des libraires les ont refusés avant de les lire. La France est le pays qui m'a accueilli, mais politiquement je suis déçu par Hollande et, sur le plan culturel, les années soixante étaient beaucoup plus riches. Il y avait des manifestations contre la guerre du Vietnam, contre le racisme, pour la défense des immigrés, choses qu'il fallait dénoncer absolument. Sartre et son équipe, Michel Foucault, ont donné à la France cet aspect de débat et de contestation. Aujourd'hui, c'est chacun pour soi. Personne ne descend dans la rue. Les plus grandes manifestations qui ont eu lieu depuis longtemps étaient pour ou contre le mariage pour tous. C'est un problème social, d'accord, mais aujourd'hui il est impossible de faire manifester les Français pour dénoncer la politique israélienne dans les territoires occupés. Personne ne descend dans la rue, au mieux ils envoient un tweet, des messages...

\section{Comment expliquez-vous ce changement ?}

C'est une question de génération, la génération après Sartre est plutôt concentrée sur ellemême que sur l'extérieur. Maintenant les débats sont à la télévision, vous avez vu le cas de Dieudonné, raciste, extrémiste. Au final, il parle de l'intégration des enfants d'immigrés, des Français, on l'a fait très mal, c'est vrai. Mais il ne m'a jamais fait rire, son humour est médiocre. De plus, il soutient les Palestiniens, mais les Palestiniens n'ont pas besoin de ce type de soutien. Il y a aussi des mouvements racistes anti-blancs, or je ne fais pas de distinction entre les hommes. Tout le monde est capable de tout. Ce n'est pas la couleur qui détermine les choses, la couleur est un hasard.

\section{Références bibliographiques}

Ben Jelloun, Tahar. L'ablation. Paris : Gallimard, 2013.

---. Le bonheur conjugal. Paris : Gallimard, 2013.

---. L'islam expliqué à ma fille. Nouvelle édition augmentée. Paris : Seuil, 2012.

---. Le racisme expliqué à ma fille. Nouvelle édition augmentée. Paris : Seuil, 2009.

---. La nuit sacrée. Paris : Seuil, 1987.

---. L'enfant de sable. Paris : Grand livre du mois, 1985. 